\title{
Peripheral and Central Pathological Mechanisms of Chronic Low Back Pain: A Narrative Review
}

\author{
Wei $\mathrm{Li}^{1}$ \\ Yinan Gong' \\ Jingyi Liu' \\ Yongming Guo ${ }^{1-3}$ \\ Huiling Tang' \\ Siru Qin' \\ Yadan Zhao' \\ Songtao Wang' \\ Zhifang $\mathrm{Xu}^{\mathrm{I}-3}$ \\ Bo Chen ${ }^{1-3}$
}

'Research Center of Experimental Acupuncture Science, Tianjin University of Traditional Chinese Medicine, Tianjin, People's Republic of China; ${ }^{2}$ School of Acupuncture \& Moxibustion and Tuina, Tianjin University of Traditional Chinese Medicine, Tianjin, 301617, People's Republic of China; ${ }^{3}$ National Clinical Research Center for Chinese Medicine Acupuncture and Moxibustion, Tianjin, 30038I, People's Republic of China

\begin{abstract}
Chronic low back pain (CLBP), lasting $>3$ months, is the end result of multiple pathogenic factors. Unfortunately, little is known about CLBP pathogenesis, which limits its advancements in clinical therapy and disease management. This paper summarizes the known pathological axes of CLBP, involving both peripheral and central systems. In particular, this paper details injurious nerve stimulation, inflammation-induced peripheral pathway, and central sensitization. Lumbar components, such as intervertebral disc (IVD), facet joints, muscles, fascia, ligaments, and joint capsules, contain pain receptors called nociceptors. Degeneration of the aforementioned lumbar components activates inflammatory pathways, which can directly damage nerves, lower nociceptor threshold to fire action potentials (AP), and cause pain. Additionally, damaged lumbar IVDs and endplates can also lead to the pathologic invasion of nerve growth and innervation, followed by the compression of herniated IVDs on nerve roots, thereby causing traumatic neuropathic pain. The central mechanism of CLBP involves alteration of the sensory processing of the brain and malfunction of the descending pain modulatory system, which facilitates pain amplification in the center nervous system (CNS). Lastly, abnormalities in the brain biochemical metabolism, activation of glial cells, and subsequent inflammation also play important roles in CLBP development. Taken together, inflammation plays an important role in both peripheral and central sensitization of CLBP. Due to the heterogeneity of CLBP, its pathological mechanism remains complex and difficult to understand. Therefore, it is a worthy field for future research into the subcomponents of CLBP pathogenesis, in order to distinguish the specific form of the disease, identify its origins, and develop corresponding highly effective comprehensive therapy against CLBP.
\end{abstract}

Keywords: chronic low back pain, inflammation, degeneration, nerve innervation, central sensitization

\section{Introduction}

Low back pain (LBP) refers to pain, muscle tension, or stiffness that occurs below the costal margin and above the inferior gluteal fold, with or without sciatica (pain that spreads from the lower back down to the legs). LBP persisting $>3$ months is termed as chronic low back pain (CLBP) and is no longer considered a symptom, but a disease caused by numerous onset factors and one that continues to progress. ${ }^{1}$ LBP is one of the most common musculoskeletal diseases among people with chronic pain, and 45-75\% of patients report feeling pain 12 months after the onset of LBP. ${ }^{2}$ Till now, no satisfactory treatments exist for LBP. A systematic review of current evidences for CLBP treatment suggests that more research is needed to fully recognize the best choice of drugs, the best drug combination, and 
the best sequence of treatments for treating CLBP. ${ }^{3}$ Although opioid analgesics are commonly used, their clinical benefits in the treatment of CLBP remain uncertain, and there is no evidence to support long-term use of any dose of opioid analgesics in the treatment of LBP. ${ }^{4}$ The efficacy evaluation of non-steroidal anti-inflammatory COX-2 inhibitor and antidepressant duloxetine in the treatment of LBP is also controversial, as it can relieve shortterm pain in some patients but has little impact on the overall improvement of back function. ${ }^{5}$ Many non-drug therapies such as exercise therapy, tai chi, yoga, psychotherapy, spinal manipulation, acupuncture, and moxibustion have also been shown to be effective in treating CLBP (strength of evidence [SOE], mild to moderate). However, few studies have reported the possibility of significant clinical improvement in CLBP. ${ }^{6}$

Between $80 \%$ and $90 \%$ of LBP patients complain of non-specific low back pain. However, since the underlying pathologic or pain-causing factors are not yet determined, the development of effective therapies is limited. ${ }^{1,7}$ Based on several ongoing investigations on the pathological mechanism(s) of CLBP, few aspects of this disease are known. For instance, most lumbar spine structures may serve as the potential origin of pain. This includes the sensory innervation in the intervertebral disc (IVD), facet joints, muscles, tendons, ligaments, fascia, synovium, joint capsule, etc. Moreover, lumbar pain can be brought on by factors such as inflammation, degeneration, or injury. Because of the high incidence of LBP, the central sensitization and systemic changes have become important pathological factors for the continuation and aggravation of LBP. Thus, this paper will systematically review the possible pathological mechanism(s) of CLBP (mainly including non-specific CLBP or radiculopathy syndromes), in terms of its peripheral and central origins, in an effort to provide detailed pathological classifications for the advancement of highly effective and targeted CLBP therapy.

\section{Peripheral Pathological Mechanism of CLBP}

\section{Peripheral Tissue Damage}

CLBP is a complex disease with high heterogeneity, and is increasingly dubbed as a mixed pain syndrome with neuropathic and injurious components. ${ }^{8}$ The peripheral tissues, such as IVDs, facet joints, muscles, tendons, ligaments, fascia, synovium, and joint capsules are rich in pain receptors called nociceptors. A series of biochemical reactions, caused by degeneration of the above mentioned tissues, can directly stimulate nociceptors, activate the nociceptive pathway, and produce pain. Similarly, a direct injury to the spinal nerve root and the pathological invasion of the nerve, due to damaged lumbar disc, could also result in neurogenic CLBP.

\section{Lumbar IVD Degeneration (IVDD)}

The IVDs are composed of internal hydrogel-like nucleus pulposus (NP), outer fibrous region-annulus fibrosus (AF), and cartilaginous endplate (CEP). The NP is a central hydrophilic gelatinous extracellular matrix (ECM) layer rich in proteoglycans. It constitutes of type II collagen and aggrecan, which are bound by a lamellated collagenous AF ring made of fibrous concentric type I collagen layers. The CEPs, on the other hand, are separate thin layered hyaline cartilages that attach IVD inferiorly and superiorly to the adjoining vertebral end plates. ${ }^{9}$ Approximately, $40 \%$ of the reported CLBP cases were found to be related to IVDD. ${ }^{10}$ IVDD is a chronic and irreversible process marked with elevated matrix degradation, NP proteoglycan loss and hydration, destruction of the disc structure (ie, loss of distinction between AF and $\mathrm{NP}$, annular tear, bulge, and prolapse), and reduced disc height. ${ }^{11,12}$ This can ultimately stimulate peripheral inflammatory cell infiltration, followed by upregulation of the levels of IL- $1 \beta$, IL- $1 \alpha$, TNF- $\alpha$, vascular and nerve growth factors, and catabolic factors. Simultaneously, matrix degradation can increase absorption of IVD tissue and activation of peripheral nerve endings, which raises peripheral nociceptors sensitization, and enhances pain sensation. ${ }^{13}$

Lumbar IVD receives sinus innervation from the dorsal root ganglion (DRG), sympathetic ganglia, and parasympathetic ganglia. ${ }^{7}$ In healthy IVD, only the outer one-third of the IVD fiber ring is innervated. However, owing to the high concentration of local neurotrophic factors (such as nerve growth factors), along with vascularized granulation, the degenerated IVD prompts the pathological vertebral nerve fibers to penetrate deeper into the inner disc (deep nerve growth), thus resulting in LBP. ${ }^{14}$ Moreover, the concentration of mechanoreceptors and calcitoninstimulated peptide-filled neurons increase in the IVD of chronic discogenic pain patients. ${ }^{15,16}$ Lastly, as the sinus nerve penetrates the nucleus pulposus, the degenerating IVD-induced inflammation further stimulates nerve discharge of the infiltrating nerve endings. ${ }^{17}$ 
CLBP patients experience more sinus nerve distribution in end plates with cartilage and subchondral bone damage, ${ }^{18}$ likely due to the chemotaxis of disc- and neovascularization-related neurotrophic factors. This could result in sensitizing sinus nerves and inducing pain. ${ }^{19,20}$ The basivertebral nerve (BVN) is a component of the sinus vertebral complex thought to play a crucial role in transmitting nociceptive information from the endplate to the spinal cord. This includes peptides like substance P, protein S-100, PGP9.5, and calcitonin gene-related peptide (CGRP) ${ }^{21-23}$ Furthermore, endplate defects can boost inflammatory cross-talks between the disc and the vertebral body, producing yet another source of pain. ${ }^{24}$ In particular, endplate defects and a drastic reduction in disc proteoglycans can augment catabolic enzymes, proinflammatory cytokines, and pro-apoptotic proteins, thereby driving IVDD. $^{25}$ In addition, an MRI study showed that an increase in the defective endplate dimensions is intimately linked to increases in disc degenerative scores, Modic changes, and disc displacement. ${ }^{26}$ Moreover, the endplate degeneration and associated changes in SI in the nucleus pulposus can surface as early as 1 year after lumbar IVDD, suggesting a faster degeneration relative to the age-dependent regression. ${ }^{27}$

Sympathetic fibers are also distributed around the annulus, endplate, vertebral body, and anterior spinal artery. In addition, a large frequency of nociceptive fibers within the IVD ring of the lower lumbar pass through the sympathetic trunk in a non-segmental manner and can be considered as part of the sympathetic nervous system (SNS). These peripheral endings display a dominant expression of calcitonin gene-related peptide, ${ }^{28,29}$ and the experimental excision of sympathetic nerves reduced pain response in patients with CLBP. ${ }^{30} \mathrm{~A}$ series of neurobiochemical reactions, initiated by IVDD, enable abundant regeneration of nociceptor and sympathetic nerve fibers, thus prompting the degenerated IVD to become more susceptible to inflammatory stimuli and pain sensation via the nociceptive transmission.

In conclusion, disc degeneration, followed by inflammation, and subsequent nociceptive fiber penetration into the inner disc, or protruding tissue mechanically pressing on the nerve root, may be the root cause of CLBP. ${ }^{8,31}$ Previous studies have reported crucial roles of nerve root injury and neuronal sensitization in the generation of chronic pain during degenerative disc disease. ${ }^{32}$ In response to inflammatory stimuli, activated macrophages in the IVD can absorb the IVD tissue and release further inflammatory mediators to stimulate the nerve roots and increase the production and sensitization of nociceptors in the nerve roots. As such, elevated afferent stimuli from sensitized nerve root nociceptors may generate augmented neuronal reactivity in the central nervous system (CNS), resulting in central sensitization. ${ }^{33}$ Likewise, peripheral sensitization within the IVD can also lead to spontaneous enhancement of pain perception. ${ }^{34}$ As a result, clinical manifestation of disc herniation involves both mechanical compression and inflammatory factors, which work in concert to produce nerve damage and sensitization, thereby leading to neuropathic pain.

\section{Facet Degeneration}

Triarticular complexes, comprised of IVD and facet joints, connect adjacent vertebrae to one another to stabilize the spine, maintain joint connections, transfer spinal load, and limit vertebral movement. ${ }^{35}$ The facet joint is a highly innervated structure consisting of the subchondral bone, articular cartilage, synovial membrane, and fibrous capsule. ${ }^{36}$ Moreover, the nerve endings originate from the medial branch that comes from the posterior ramus and are responsible for mostly pain and proprioception signals from the middle of the spine to the facet joint. ${ }^{37}$ Facet pain accounts for 16-40\% of the CLBP cases and it increases with age. ${ }^{38,39}$ The osteoarthritic facet joints or buildup of capsule pressure in facet joints may promote sensitization of nociceptors and, together with fat-induced spinal nerve injury, ${ }^{40}$ produce pain sensation. ${ }^{41}$ However, the specific mechanism is still under investigation.

\section{Degeneration of the Lumbar Muscle Fascia}

A healthy spine requires optimal performance of the back muscles. The core muscles of the lumbar spine, namely the lumbar multifidus and erector spinae, stabilize the spinal formation. Therefore, any structural alterations in these muscles contribute to functional limitations. Additionally, multiple studies demonstrated that persistent CLBP is typically associated with muscle structural changes, as evidenced by alterations in motion patterns to safeguard the deep multifidus from excess load, ${ }^{42}$ pain/fear, and avoidance of movement. ${ }^{43,44}$ One mechanism of muscle structural change in CLBP patients could be arthrogenous muscle inhibition, which is a process where a localized reflex inhibition is caused due to joint pain and weakened driving force of muscle and nerve stabilizing the joint. ${ }^{45}$ There are reports of CLBP patients suffering from spinal joint muscle suppression and electromyographic (EMG) 
evidence revealed that the patients with back pain have weakened neural drive to the multifidus muscle (MF). ${ }^{46}$ Moreover, in healthy volunteers and in patients with CLBP, it is reported that pain reduced not only the neural drive of MF, but also the nerve drive of the erector spine muscle. ${ }^{47}$ Nociceptive or pain signals from the spine can inhibit the neuromuscular control system in the brain and spinal cord, resulting in decreased muscular nerve drive and a reduced stability and movement of the spine. Impaired neural drives also alter muscular proprioceptive feedback signals. ${ }^{48}$ As a result, patients with recurrent LBP (RLBP) often experience recombination of trunk muscle performance associated with postural control defects in their motor cortex. ${ }^{49}$

It was previously reported that the fat infiltration into the paraspinal muscle was the most relevant factor, rather than the IVDD and end-plate alterations, in generating LBP in women. ${ }^{50}$ And the most commonly reported cause of LBP was severe IVDD, which is closely related to the end-plate changes (Modic changes) and fat infiltration into the multifidus and erector spinae of the corresponding lumbar vertebrae. ${ }^{51}$ In addition, long-term alterations in motion patterns in CLBP patients can ultimately result in structural changes within muscle fibers. It was previously reported that increased neuromuscular activity or massive mechanical load promotes the formation of slow-oxidizing muscle fibers, whereas decreased neuromuscular activity prompts generation of fastglycolytic muscle fibers. ${ }^{52}$ As a result, RLBP patients exhibit muscle deterioration, which worsens with more frequent or more persistent back pain. Relative to discontinuous CLBP and RLBP, persistent CLBP patients experience increased adipose infiltration, reduced muscle quality, and diminished muscle efficiency. ${ }^{53}$ The frequency of pain may also alter the metabolic activity of the lower back muscles. As a result, glycolytic or anaerobic fibers (type II fibers) are more commonly detected in the back muscles of CLBP patients, ${ }^{54}$ along with more metabolite generation during contraction. ${ }^{55}$ Moreover, muscle biopsy from LBP patients exhibits a reduced number of type I fibers with low fatigue resistance. ${ }^{56}$ Alternately, the amount of type II and intermediate IIc fibers remain high indicating ongoing fiber conversion from a highly efficient muscle type to one that uses anaerobic respiration to produce short bursts of mobility. ${ }^{57}$ Moreover, persistent pain and inflammation can give rise to additional muscle dysregulation, such as, atrophy, fatty infiltration, decreased strength/endurance, and loss of function. In a vicious feedback loop, these complex bidirectional interrelationships often drive recurring or persistent LBP cycles. ${ }^{58,59}$ Interestingly, there are also reports of the non-specific chronic low back pain (NSCLBP) patients, harboring paraspinal muscles with a larger number of type I fibers and lower number of type IIx glycolytic fibers, thereby increasing their oxidative potential. ${ }^{60}$ This may be due to the increased mechanical load on the paraspinal muscle due to a shift in gait in NSCLBP patients.

Furthermore, along with muscle nociceptors, animal studies have also identified muscle fascia as yet another source of nociception. ${ }^{61}$ Inflammatory nodules activate muscle nociceptors during skeletal muscle tension. ${ }^{62}$ Moreover, myofascial trigger points may lead to primary hyperalgesia under sustained noxious stimuli. ${ }^{63}$ As a result, tissue near the myofascial trigger points become acidic and exhibit higher levels of substance P, CGRP, tumor necrosis factor, and IL-1, each of which contributes to increased pain sensitivity. ${ }^{64}$

\section{Role of Peripheral Inflammation in CLBP}

Inflammation plays a major role in the pathogenesis of CLBP; namely, in the degeneration of disc, endplate, facet joints, and pathological processes of muscle fascia, nerve, and other tissue. As such, inflammation is also presumed to be involved in the pathogenesis of CLBP and related pain.

\section{Degenerative Inflammation of the Disc}

Multiple prior reports suggest that both IVD resident cells (NP and annulus fibrosus) and non-resident cells (macrophages) produce a variety of pro-inflammatory molecules after IVDD or damage. ${ }^{65,66}$ Moreover, these cells are reported to contribute to the inflammatory etiology of IVDD. ${ }^{7}$ At the onset of the IVD degenerating cascade, the highly vascularized AF and NP enable mast cells and macrophages to migrate to the disc, and exacerbate inflammation within the disc, and produce LBP. ${ }^{67}$ The role of macrophages in other forms of neuropathic and inflammatory pain is well established. In particular, they are known to infiltrate damaged nerves and dorsal root ganglia through phagocytosis, inflammatory mediator secretion, and angiogenesis. ${ }^{13,68}$ This infiltration process has been detected in both human and rodent regressive IVDs cases. $^{66,69}$ Moreover, the upregulated expression of IL$1 \beta$, IL-1 $\alpha, \mathrm{TNF}-\alpha$, vascular endothelial growth factor (VEGF) and its receptor, and basic fibroblast growth factor (BFGF) in degenerated disc tissue, along with enhanced 
neovascularization accelerates catabolism, reduces proteoglycan production, and enhances matrix metalloproteinase expression. ${ }^{70}$ Proteoglycans prevent growth of blood vessels and nerves to the NP. Excessive metalloproteinase activation and matrix degradation can stimulate the growth of in-disc blood vessels and nerves ${ }^{71}$ thereby activating and sensitizing sensory nerve fibers. ${ }^{72}$ Increased levels of nerve growth factor (NGF), brain-derived neurotrophic factor (BDNF) and inflammation can activate sprouting of the DRG nerve fibers into the AF and NP, which increases neural survival in DRG and augments nociceptive cation channel sensitivity, and ultimately induces pain. ${ }^{67,70}$ Based on several studies, artificially suppressing the nociceptive factors and pro-inflammatory mediators, secreted by degenerative discs, can reduce the nociceptive input and inactivate the discs. Furthermore, suppressing TLR4 also reduces signs of disc degeneration and pain, as evidenced in SPARC-deficient mice. ${ }^{73}$

Inflammation is another factor regulating regression of lumbar disc herniation (LDH). In fact, inflammation and neovascularization can induce phagocytic enzyme-related degradation and spontaneous absorption of herniated disc tissue. $^{65,74}$ Inflammation is triggered when disc contents are squeezed into the epidural space. Additionally, the role of macrophages is crucial for this process. Multiple studies have detected the presence of macrophages in herniated IVD tissue specimens using immunohistochemistry. ${ }^{75}$ These cells actively engulf herniated tissues and process themselves in lysosomes filled with collagen-degrading enzymes. Macrophages are also known to secrete lysosomal enzymes through exocytosis and contribute to the intercellular breakdown of substances, such as the IVD matrix components proteoglycan and collagen. ${ }^{76}$ In addition, IVD cells can produce inflammatory mediators, ${ }^{77}$ which may help to recruit other immune cells to herniated tissues, namely, monocyte chemoattractant protein (MCP)1, a CC chemokine responsible for facilitating monocyte activation and recruitment. ${ }^{65}$ Under physiological conditions, a mature IVD is lightly vascularized. However, neovascularization is reported at the edge of protruding tissue and is the main determinant of the spontaneous regression of $\mathrm{LDH}^{74}$ Taken together, inflammation plays a critical role in lumbar IVDD. With the infiltration of immune cells into the IVD, neovascularization, nerve growth, IVD nerve compression, nerve sensitization, and inward growth can all work in concert to excessively stimulate nociceptive receptors and induce LBP.

\section{Inflammation in Peripheral Circulation}

Inflammatory response is also detected in the peripheral blood of CLBP patients, presenting M1-type monocyte dominance, imbalance between pro-inflammatory and anti-inflammatory cytokines, down-regulation of IL-10 levels, up-regulation of IL-6 levels, and decrease in opioid secretin ability of M2-type macrophages. ${ }^{78}$ Moreover, pro-inflammatory biomarkers like C-reactive protein (CRP) and IL-6 are shown to be positively associated with the severity of non-specific low back pain (NSLBP), while TNF-a is positively related to the presence of NSLBP. ${ }^{79}$ Using preclinical models, it was revealed that a rise in IL-6 enhances TNF-a generation in DRG neurons and accelerates hyperalgesia. ${ }^{80}$ Moreover, leptin and MCP-1 are reported to be biomarkers marking the transition of acute LBP into chronic disease. In addition, alterations in plasma N-glycation levels in CLBP patients are consistent with common n-glycation changes during chronic inflammation. ${ }^{81}$ Based on these reports, systemic and/or local inflammatory processes are partly responsible for the progression of CLBP in LBP patients.

\section{Central Pathological Mechanism of CLBP}

Recent evidence suggests that the nociceptive mechanism of central sensitization, including neuronal hyperactivity in the CNS may contribute to the persistent pain in CLBP, in the absence of noxious stimuli. ${ }^{82-84}$ Based on an epidemiology study, there was a high concentration of trigger points (TrPs) in the psoas muscle of CLBP patients, relative to those without CLBP. ${ }^{85}$ Moreover, it was shown that prolonged nociceptive input from TrPs can alter brain plasticity and drive the development and maintenance of chronic musculoskeletal pain. ${ }^{86,87}$ Therefore, central pathological changes may be an important factor in the pathogenesis of CLBP.

\section{Abnormalities in the Central Pain Modulation System}

Along with the pain mechanisms involving peripheral nociceptive and nerve root injury, the central pain modulation system can also be a major factor in the development of CLBP. ${ }^{88}$ CLBP patients have lower stress pain threshold, compared to healthy people. ${ }^{89}$ This may be due to the stimulation of the nociceptive pathway that expands the receptive field of the dorsal horn of the spinal cord, excites 
associated pain regions in the CNS, and lowers the pain threshold based on the duration and intensity of the pain syndrome, ${ }^{90}$ thereby sensitizing the entire spinal cord segment that interacts with muscles, ligaments, and skin. ${ }^{91}$ Recently, multiple brain imaging studies investigated brain structural and functional changes in relation to CLBP. Based on these studies, CLBP patients display characteristic features in the sensorimotor systems, ${ }^{92}$ attention network, ${ }^{93}$ default mode network, ${ }^{93}$ and pain modulation network, such as the structural and functional changes of the pain modulation system, ${ }^{94}$ relative to healthy controls. According to a systematic review, CLBP patients exhibit enhanced activity in certain cortical and subcortical areas, such as higher activation of the medial PFC, cingulate cortex, amygdala, and insular lobe, and reduced activity in the pain-relief areas and altered functional connectivity (FC) in pain-associated areas. ${ }^{95}$ These findings are indicative of a relationship between CLBP and broad changes in brain networks. Conditioned pain modulation (CPM) is one of the methods used to evaluate the function of endogenous descending pain modulation system. ${ }^{96}$ The neuroanatomical basis of CPM includes brainstem structures like periaqueductal gray (PAG) that is regulated by both serotonergic and non-adrenergic systems. Using highintensity nociceptors-mediated stimulation of the brainstem, neuronal impulses from the spinal cord can be suppressed, resulting in an anti-irritating low-polarization response, which ultimately results in extensive hypoalgesia. ${ }^{96}$ Similarly, a meta-analysis discovered that LBP patients exhibit severe CPM damage followed by a remarkable rise in the temporal summation of pain. In fact, chronic and severe pain has the greatest influence on CPM between LBP patients and healthy controls. ${ }^{97}$ CLBP patients typically exhibit abnormal FC in the central PAG pain modulation at rest. ${ }^{94}$ In addition, the $\mathrm{FC}$ between PAG and ventral prefrontal cortex (VmPFC)/rostral anterior cingulate gyrus (RACC) is enhanced in CLBP patients. Lastly, a significant negative correlation between pain score and PAG-vmPFC/rACC FC is reported in CLBP patients. These findings are consistent with the descending pain modulation dysfunction in CLBP patients. ${ }^{94}$

The transition from acute LBP to CLBP is also accompanied by changes in the CNS. One study found that the individuals with persistent pain exhibit increased FC between the medial prefrontal cortex (mPFC) and the nucleus accumbens (NAC) at initial evaluation. ${ }^{98}$ After 3 years, the structural components associated with pain, along with FC between mPFC, amygdale, and NAC, altered in persistent LBP and those who recovered from LBP. However, those who progressed to chronic pain had relatively smaller amygdala and hippocampus volumes, suggesting that these structural changes may have occurred before the onset of pain, potentially predisposing individuals to develop chronic pain. ${ }^{99}$ In addition, cognitive and emotional responses to pain can also be important factors in the development and maintenance of chronic pain. ${ }^{100}$ With a shift to chronic pain, brain activity, associated with back pain perception, shifts from areas involved in acute pain to areas participating in emotional circuits such as medial prefrontal cortex/amygdale. As a result, the perception shifts from pain-oriented to specific emotions such as fear, anger and sadness. ${ }^{101}$ Therefore, acute/sub-acute back pain is regulated by both pain and reward circuits. Multiple reports validate that in CLBP and in late persistent LBP (after 6-12 months of back pain) patients, there is a gradual decrease in the participation of acute pain circuits, and a simultaneous increase in the involvement of emotional and reward circuits. ${ }^{101}$ Moreover, once the CLBP or late persistent LBP brain characteristics develop, they remain stable and unchanged for the next 10 years. ${ }^{101}$ This study suggests that the brain forms a state of chronic pain within the first year of LBP. Therefore, the first year is a critical period for brain alterations in back pain patients. Moreover, this period remains consistent with the clinical definition of the transition standard pain to chronic pain and is assumed to be between 3 and 12 months.

\section{Central Inflammation}

Using integrated positron emission tomography (ICT-MRI) and radioactive oligonucleotide C-PBR28, elevated levels of glial cell activation marker (translocator protein) were found in the brains of CLBP patients. ${ }^{102}$ Along with glial cell activation, cytokines induced by neuroinflammation in the CNS, may also target pain and hyperalgesia. ${ }^{103,104}$ In fact, a positive correlation is reported between IL-8 levels in serum and cerebrospinal fluid (CSF) of patients with chronic lumbar disc herniation (LDH). Moreover, increase in CSF IL-8 is correlated with pain intensity and pain sensitivity of the vertebral pressure, suggesting an IL8-mediated neuroimmune crosstalk regulating neuroinflammation and pain sensitivity in LDH patients. Additionally, MCP1 is known to promote differentiation of activated microglia, ${ }^{103,104}$ increase blood-brain barrier (BBB) permeability via MCP1 receptors, ${ }^{105}$ participate in crosstalk 
between the peripheral and the $\mathrm{CNS},{ }^{106}$ and produce large quantities of IL-8 and MCP1 via the activated glial cells. ${ }^{107,108}$ Subsequently, the peripheral T cells and monocytes pass through the $\mathrm{BBB}$ and travel to the spinal cord parenchyma, resulting in massive neuroimmune activity. Lastly, these inflammatory responses send signals to the brain and, in a vicious feedback loop, perpetuate more inflammation of neurons in the CNS. ${ }^{33,109}$

A number of inflammatory biomarkers like cystatin $\mathrm{C}$, neural cell adhesion molecule L1-like protein, and amyloid-like protein 1 were found to be elevated in CSF of IVDD patients, regardless of the state of pain. CLBP patients experiencing IVDD-related neuropathy often show a rise in the levels of hemagglutinin, various apolipoproteins, insulin-like growth factor II and fibronectin, which are induced by nerve injury. Conversely, asymptomatic IVDD patients only show changes in the CSF cystatin C levels. ${ }^{110}$ The differences in the presence of CSF proteins in these two conditions may be attributed to the destruction of the blood-spinal cord barrier ${ }^{111}$ and $\mathrm{BBB}^{112}$ after nerve injury, which allows peripheral molecules and white blood cells to enter CSF. It may also be due to a state of nerve injury-related edema that alters the flow of molecules from the injured nerve to the CSF. In all, the changes in CSF proteins reflect the peripheral regulatory pathways of CNS, such as central sensitization and neuroinflammation. ${ }^{13}$

\section{Discussion}

In summary, the pathology of CLBP is heterogeneous. Taking both CLBP peripheral and central pathological mechanisms into account, the pathogenesis potentially includes peripheral nociceptive stimulation, peripheral and/or central inflammation, central pain modulation network abnormality, and a mixed onset of various pathological mechanisms. In short, lumbar and pelvic spinal components, such as IVD, muscle, fascia, facet joint, sacroiliac joint, pubic symphysis, ligament and joint capsule contain nociceptors. Any event that brings about tissue degeneration activates massive inflammatory response that infiltrates the IVD, joint, muscle, fascia, and other tissues, stimulates nociceptive receptors to produce inflammatory substances, which directly damages the nerve root, and generates pain. Simultaneously, the injured lumbar IVD and endplate stimulate pathological and invasive nerve growth and distribution, followed by the compression of the nerve root by IVD herniation tissue, thereby causing neuropathic pain. Growing evidences also point to inflammation playing a critical role in the pathogenesis of CLBP. The central mechanism of LBP involves altering sensory processing in the brain, ${ }^{83}$ such as the dysfunction of descending pain modulation system, ${ }^{94}$ resulting in the amplification of pain information in the brain. Recently, abnormal biochemical metabolism and activation of glial cells in the brain were also shown to play essential roles in CLBP.

Although there is some understanding of the pathogenesis of CLBP, more specific and reliable investigations are warranted for a comprehensive analysis into the intricate details of the complex CLBP pathogenesis. For instance, the role of small joints in the development of LBP is still unclear. Moreover, there are few studies on central inflammation and glial cell activation and its involvement in the peripheral inflammation in CLBP patients. However, the exact mechanism and its contribution to the development of CLBP still remains to be understood.

Given the heterogeneous nature of CLBP pathology, it is either possible that one mechanism gives rise to all the disruptions seen peripherally and centrally or a number of dysregulations result in the above-mentioned disruptions and result in CLBP. Hence, it is shown that CLBP can be injurious, neurotic, or both. It is possible that the local inflammation of the lower back induces peripheral inflammation, which reduces the pain-relieving opioid secreting ability of peripheral blood cells in CLBP patients, resulting in pain. Previous studies have reported that the presence of neuropathic components was associated with a greater intensity and a longer duration of pain, with higher prevalence in patients with comorbidities. ${ }^{113}$ Many treatments are available for CLBP; however, conclusions from several clinical studies remain inconclusive and the level of evidence is low. One such CLBP therapy is COX-2 inhibitor, which was considered a first-line NSAIDs treatment for CLBP in a systematic review. ${ }^{114}$ However, a recent trial revealed a smaller benefit of NSAIDs, as compared to placebo. ${ }^{115}$ The unclear heterogeneous pathology of CLBP may be to blame for this discrepancy. In a meta-analysis exploring the efficacy of CLBP therapy, the trials differed significantly in their patient selection and may have accidentally included patients with neuropathic pain. Neuropathic pain may, sometimes, exist in the absence of local neurological findings and may, therefore, be mistakenly assigned as CLBP. Moreover, different pain conditions may be affected by the same drug, thereby influencing the combined estimate of the level of therapeutic effect. ${ }^{114}$ Therefore, to clarify the 
pathological mechanism of CLBP, it is important to conduct subcomponent investigation to reduce heterogeneity of CLBP, improve homogeneity of the disease, and provide effective clinical diagnosis and treatment. Similar concerns have been raised in academia that exploring the pathogenesis of NSLBP may be too broad of a concept to tackle together. And existing NSLBP therapy use only generic drugs to treat pain and its consequences. Although this therapy is effective to a certain extent, it is moderate at best. Therefore, the next approach to examining LBP pathology is to determine LBP phenotype based on the pathological anatomy or through clinical reasoning. ${ }^{116}$ Researchers and clinicians have explored the subgroup classification of LBP in an attempt to subdivide LBP patients into homogeneous populations with similar characteristics to improve patient outcomes. A systematic review shows that current efforts to explore the classification of LBP subset patients have focused on the following five attributes: (I) clinical characteristics; (II) pathological anatomy; (III) treatment-based approaches; (IV) screening tools and prediction rules; and (V) pain mechanisms. ${ }^{117}$ However, research is still ongoing and there is still no consensus on the current classification standards. The reliability of the subgroup effects was found to be quite low in the systematic review. Moreover, there was insufficient evidence to support the subgroup classification, owing to the inadequate statistical analysis or contradictory authors' claims. ${ }^{118}$

Furthermore, due to the complex and heterogeneous pathologic mechanism of CLBP, monotherapy may not achieve ideal results. In fact, several studies have proposed that the most reasonable therapy should be a multi-mode approach integrating multi-disciplinary treatment and coordinating somatic and psychological therapeutic elements. Moreover, in a multi-level approach, individual combination therapy can improve the analgesic effect and reduce the dosage of drugs, thus reducing the incidence of side effects. ${ }^{113}$ Global clinical practice guidelines use comprehensive management of CLBP patients, including education and comfort, analgesics, non-drug therapy, and timely response based on the individual patient's needs, possible prognosis, and attention to serious pathological abnormalities. ${ }^{116}$ In addition, compared to treating acute LBP, persistent CLBP therapy should place more emphasis on non-drug therapy, and more consideration on the treatment of coexisting diseases such as depression. This is further illustrated in several systematic reviews evidencing lack of effective therapy and the need for research into the best choice of therapeutic agents, the best combination and sequential treatment, as well as the most effective drug in the treatment of $\mathrm{LBP}^{3}$ As mentioned before, many non-drug therapies such as exercise therapy, Tai chi, yoga, psychotherapy, spinal manipulation, and acupuncture have shown some efficacy in CLBP management (SOE, mild to moderate), but few studies have reported clinically significant improvement. Since monotherapy is not optimal, ${ }^{5}$ it is necessary to advance comprehensive management recommended by the current international clinical guidelines and reasonably combine multiple therapies to improve the clinical effect of CLBP therapy.

Moreover, due to the lack of effective CLBP therapy, opioids are still widely used to manage pain. However, its repeated and long-term use may promote tolerance (desensitization to opioid drugs) and, therefore, make it ineffective for future pain management. Additionally, long-term opioid use may develop opioid dependence, which can cause undesirable withdrawal syndromes, such as, agitation, insomnia, diarrhea, and hyperalgesia. ${ }^{119}$ Finally, due to extensive research into the pathological understanding of CLBP, much is known about the disease than ever before. However, more investigations are warranted to gain a comprehensive understanding of all intricate pathways involved in CLBP pathogenesis. These investigations should specifically target the subcomponents of CLBP in order to promote highly effective and integrated management therapy for CLBP.

\section{Abbreviations}

CLBP, chronic low back pain; IVD, intervertebral disc; AP, action potentials; CNS, center nervous system; LBP, low back pain; SOE, strength of evidence; NP, nucleus pulposus; AF, annulus fibrosus; CEP, cartilaginous endplate; ECM, extracellular matrix; IVDD, IVD degeneration; DRG, dorsal root ganglion; BVN, basivertebral nerve; CGRP, calcitonin gene-related peptide; SNS, sympathetic nervous system; EMG, electromyographic; MF, multifidus muscle; RLBP, recurrent LBP; NSCLBP, nonspecific chronic low back pain; VEGF, vascular endothelial growth factor; BFGF, basic fibroblast growth factor; NGF, nerve growth factor; BDNF, brain-derived neurotrophic factor; LDH, lumbar disc herniation; MCP, monocyte chemoattractant protein; CRP, C-reactive protein; TrPs, trigger points; FC, functional connectivity; CPM, conditioned pain modulation; $\mathrm{PAG}$, periaqueductal gray; VPFC, ventral prefrontal cortex; RACC, rostral anterior cingulate gyrus; mPFC, medial prefrontal cortex; NAC, 
nucleus accumbens; CSF, cerebrospinal fluid; LDH, lumbar disc herniation; BBB, blood-brain barrier.

\section{Acknowledgments}

The authors acknowledge financial support from the National Natural Science Foundation of China (NSFC) and China Association for Science and Technology.

\section{Disclosure}

This research was supported by grants from the National Key R\&D Program of China No. 2019YFC1709003, the Youth Talent Promotion Project of China Association for Science and Technology (2019-2021ZGZJXH-QNRC001), the National Natural Science Foundation of China (NSFC) No. 82074534, and the Scientific research projects of Tianjin Education Commission (2017KJ163). The authors declare that they have no competing interest.

\section{References}

1. Vlaeyen JWS, Maher CG, Wiech $\mathrm{K}$, et al. Low back pain. Nat Rev Dis Primers. 2018;4(1):53.

2. Hestbaek L, Leboeuf-Yde C, Manniche C. Low back pain: what is the long-term course? A review of studies of general patient populations. Eur Spine J. 2003;12(2):149-165. doi:10.1007/ s00586-002-0508-5

3. Chou R, Deyo R, Friedly J, et al. Systemic pharmacologic therapies for low back pain: a systematic review for an American college of physicians clinical practice guideline. Ann Intern Med. 2017;166(7):480-492. doi:10.7326/M16-2458

4. Abdel Shaheed C, Maher CG, Williams KA, Day R, McLachlan AJ. Efficacy, tolerability, and dose-dependent effects of opioid analgesics for low back pain: a systematic review and meta-analysis. JAMA Intern Med. 2016;176(7):958-968. doi:10.1001/jamainternmed.2016.1251

5. Chou R, Deyo R, Friedly J, et al. Nonpharmacologic therapies for low back pain: a systematic review for an American college of physicians clinical practice guideline. Ann Intern Med. 2017;166 (7):493-505. doi:10.7326/M16-2459

6. Delitto A, George SZ, Van Dillen L, et al. Low back pain. J Orthop Sports Phys Ther. 2012;42(4):A1-A57. doi:10.2519/ jospt.2012.42.4.A1

7. Ohtori S, Inoue G, Miyagi M, Takahashi K. Pathomechanisms of discogenic low back pain in humans and animal models. Spine J. 2015;15(6):1347-1355. doi:10.1016/j.spinee.2013.07.490

8. Freynhagen R, Baron R. The evaluation of neuropathic components in low back pain. Curr Pain Headache Rep. 2009;13 (3):185-190. doi:10.1007/s11916-009-0032-y

9. Mirza SK, White AA. Anatomy of intervertebral disc and pathophysiology of herniated disc disease. J Clin Laser Med Surg. 1995;13(3):131-142. doi:10.1089/clm.1995.13.131

10. Andersson GB. Epidemiological features of chronic low-back pain. Lancet. 1999;354(9178):581-585. doi:10.1016/S01406736(99)01312-4

11. De Geer CM. Cytokine involvement in biological inflammation related to degenerative disorders of the intervertebral disk: a narrative review. J Chiropr Med. 2018;17(1):54-62. doi:10.1016/j.jcm.2017.09.003
12. Sampara P, Banala RR, Vemuri SK, Av GR, Gpv S. Understanding the molecular biology of intervertebral disc degeneration and potential gene therapy strategies for regeneration: a review. Gene Ther. 2018;25(2):67-82. doi:10.1038/s41434-018-0004-0

13. Scholz J, Woolf CJ. The neuropathic pain triad: neurons, immune cells and glia. Nat Neurosci. 2007;10(11):1361-1368. doi:10.1038/nn1992

14. Freemont AJ, Peacock TE, Goupille P, Hoyland JA, O'Brien J, Jayson MI. Nerve ingrowth into diseased intervertebral disc in chronic back pain. Lancet. 1997;350(9072):178-181. doi:10.1016/S0140-6736(97)02135-1

15. Roberts S, Eisenstein SM, Menage J, Evans EH, Ashton IK. Mechanoreceptors in intervertebral discs. Morphology, distribution, and neuropeptides. Spine. 1995;20(24):2645-2651. doi:10.1097/00007632-199512150-00005

16. Morinaga T, Takahashi K, Yamagata M, et al. Sensory innervation to the anterior portion of lumbar intervertebral disc. Spine. 1996;21(16):1848-1851. doi:10.1097/00007632-19960815000002

17. Simon J, McAuliffe M, Shamim F, Vuong N, Tahaei A Discogenic low back pain. Phys Med Rehabil Clin $N$ Am. 2014;25(2):305-317. doi:10.1016/j.pmr.2014.01.006

18. Fields AJ, Liebenberg EC, Lotz JC. Innervation of pathologies in the lumbar vertebral end plate and intervertebral disc. Spine J. 2014;14(3):513-521. doi:10.1016/j.spinee.2013.06.075

19. Freemont AJ, Watkins A, Le Maitre C, et al. Nerve growth factor expression and innervation of the painful intervertebral disc. J Pathol. 2002;197(3):286-292. doi:10.1002/path.1108

20. García-Cosamalón J, Del Valle ME, Calavia MG, et al. Intervertebral disc, sensory nerves and neurotrophins: who is who in discogenic pain. J Anat. 2010;217(1):1-15. doi:10.1111/ j.1469-7580.2010.01227.x

21. Ohtori $\mathrm{S}$, Inoue $\mathrm{G}$, Ito $\mathrm{T}$, et al. Tumor necrosis factor-immunoreactive cells and PGP 9.5-immunoreactive nerve fibers in vertebral endplates of patients with discogenic low back pain and modic type 1 or type 2 changes on MRI. Spine. 2006;31 (9):1026-1031. doi:10.1097/01.brs.0000215027.87102.7c

22. Kumar N, Kumar A, Siddharth MS, Sambhav PS, Tan J. Annulonucleoplasty using Disc-FX in the management of lumbar disc pathology: early results. Int $J$ Spine Surg. 2014;8:18. doi: $10.14444 / 1018$

23. Choi SH, Adsul N, Kim HS, Jang JS, Jang IT, Oh SH. Magnetic resonance imaging undetectable epiduroscopic hotspot in chronic diskogenic back pain-does sinuvertebral neuropathy actually exist. World Neurosurg. 2018;110:354-358. doi:10.1016/j. wneu.2017.11.151

24. Dudli S, Sing DC, Hu SS, et al. ISSLS PRIZE IN BASIC SCIENCE 2017: intervertebral disc/bone marrow cross-talk with modic changes. Eur Spine J. 2017;26(5):1362-1373. doi:10.1007/ s00586-017-4955-4

25. Lotz JC, Fields AJ, Liebenberg EC. The role of the vertebral end plate in low back pain. Global Spine J. 2013;3(3):153-164. doi:10.1055/s-0033-1347298

26. Zehra U, Cheung J, Bow C, Lu W, Samartzis D Multidimensional vertebral endplate defects are associated with disc degeneration, modic changes, facet joint abnormalities, and pain. J Orthop Res. 2019;37(5):1080-1089. doi:10.1002/ jor. 24195

27. Luoma K, Vehmas T, Kerttula L, Grönblad M, Rinne E. Chronic low back pain in relation to modic changes, bony endplate lesions, and disc degeneration in a prospective MRI study. Eur Spine J. 2016;25(9):2873-2881. doi:10.1007/s00586-016-4715-x

28. Brown MF, Hukkanen MV, McCarthy ID, et al. Sensory and sympathetic innervation of the vertebral endplate in patients with degenerative disc disease. J Bone Joint Surg Br. 1997;79 (1):147-153. doi:10.1302/0301-620X.79B1.0790147 
29. Aoki Y, Ohtori S, Takahashi K, et al. Innervation of the lumbar intervertebral disc by nerve growth factor-dependent neurons related to inflammatory pain. Spine. 2004;29(10):1077-1081. doi:10.1097/00007632-200405150-00005

30. Murata Y, Olmarker K, Takahashi I, Takahashi K, Rydevik B. Effects of lumbar sympathectomy on pain behavioral changes caused by nucleus pulposus-induced spinal nerve damage in rats. Eur Spine J. 2006;15(5):634-640. doi:10.1007/s00586-0051020-5

31. Cohen SP, Mao J. Neuropathic pain: mechanisms and their clinical implications. BMJ. 2014;348:f7656. doi:10.1136/bmj.f7656

32. Pezet S, McMahon SB. Neurotrophins: mediators and modulators of pain. Annu Rev Neurosci. 2006;29:507-538. doi:10.1146/ annurev.neuro.29.051605.112929

33. Hunt JL, Winkelstein BA, Rutkowski MD, Weinstein JN, DeLeo JA. Repeated injury to the lumbar nerve roots produces enhanced mechanical allodynia and persistent spinal neuroinflammation. Spine. 2001;26(19):2073-2079. doi:10.1097/ 00007632-200110010-00005

34. Shiri R, Falah-Hassani K, Viikari-Juntura E, Coggon D. Leisuretime physical activity and sciatica: a systematic review and meta-analysis. Eur J Pain. 2016;20(10):1563-1572. doi:10.1002/ ejp. 885

35. Jaumard NV, Welch WC, Winkelstein BA. Spinal facet joint biomechanics and mechanotransduction in normal, injury and degenerative conditions. J Biomech Eng. 2011;133(7):071010. doi:10.1115/1.4004493

36. Kallakuri S, Li Y, Chen C, Cavanaugh JM. Innervation of cervical ventral facet joint capsule: histological evidence. World J Orthop. 2012;3(2):10-14. doi:10.5312/wjo.v3.i2.10

37. Bogduk N, Long DM. The anatomy of the so-called "articular nerves" and their relationship to facet denervation in the treatment of low-back pain. J Neurosurg. 1979;51(2):172-177. doi:10.3171/ jns.1979.51.2.0172

38. Manchikanti L, Singh V, Pampati V, Damron KS, Beyer CD, Barnhill RC. Is there correlation of facet joint pain in lumbar and cervical spine? An evaluation of prevalence in combined chronic low back and neck pain. Pain Physician. 2002;5 (4):365-371. doi:10.36076/ppj.2002/5/365

39. Manchikanti L, Pampati V, Rivera J, Fellows B, Beyer C, Damron K. Role of facet joints in chronic low back pain in the elderly: a controlled comparative prevalence study. Pain Pract. 2001;1(4):332-337. doi:10.1046/j.1533-2500.2001.01034.x

40. Bogduk N. Evidence-informed management of chronic low back pain with facet injections and radiofrequency neurotomy. Spine $J$. 2008;8(1):56-64. doi:10.1016/j.spinee.2007.10.010

41. Genevay S, Atlas SJ. Lumbar spinal stenosis. Best Pract Res Clin Rheumatol. 2010;24(2):253-265. doi:10.1016/j.berh.2009.11.001

42. Claus AP, Hides JA, Moseley GL, Hodges PW. Different ways to balance the spine in sitting: muscle activity in specific postures differs between individuals with and without a history of back pain in sitting. Clin Biomech. 2018;52:25-32. doi:10.1016/j. clinbiomech.2018.01.003

43. Karayannis NV, Smeets RJ, van den Hoorn W, Hodges PW. Fear of movement is related to trunk stiffness in low back pain. PLoS One. 2013;8(6):e67779. doi:10.1371/journal.pone.0067779

44. Mannion AF, Käser L, Weber E, Rhyner A, Dvorak J, Müntener M. Influence of age and duration of symptoms on fibre type distribution and size of the back muscles in chronic low back pain patients. Eur Spine J. 2000;9(4):273-281. doi:10.1007/s005860000189

45. Rice DA, McNair PJ. Quadriceps arthrogenic muscle inhibition: neural mechanisms and treatment perspectives. Semin Arthritis Rheum. 2010;40(3):250-266. doi:10.1016/j.semarthrit. 2009.10.001
46. D'hooge R, Hodges P, Tsao H, Hall L, Macdonald D, Danneels L. Altered trunk muscle coordination during rapid trunk flexion in people in remission of recurrent low back pain. $J$ Electromyogr Kinesiol. 2013;23(1):173-181. doi:10.1016/j.jelekin.2012.09.003

47. Dubois JD, Piché M, Cantin V, Descarreaux M. Effect of experimental low back pain on neuromuscular control of the trunk in healthy volunteers and patients with chronic low back pain. $J$ Electromyogr Kinesiol. 2011;21(5):774-781. doi:10.1016/j. jelekin.2011.05.004

48. Wand BM, Parkitny L, O'Connell NE, et al. Cortical changes in chronic low back pain: current state of the art and implications for clinical practice. Man Ther. 2011;16(1):15-20. doi:10.1016/j. math.2010.06.008

49. Tsao H, Galea MP, Hodges PW. Reorganization of the motor cortex is associated with postural control deficits in recurrent low back pain. Brain. 2008;131(Pt 8):2161-2171. doi:10.1093/ brain/awn154

50. Özcan-ekşi EE, Mş E, Turgut VU, et al. Reciprocal relationship between multifidus and psoas at L4-L5 level in women with low back pain. $B r J$ Neurosurg. 2020.

51. Özcan-ekşi EE, Ekşi MŞ, Akçal MA. Severe lumbar intervertebral disc degeneration is associated with modic changes and fatty infiltration in the paraspinal muscles at all lumbar levels, except for L1-L2: a cross-sectional analysis of 50 symptomatic women and 50 age-matched symptomatic men. World Neurosurg. 2019;122:e1069-e1077. doi:10.1016/j.wneu.2018.10.229

52. Baldwin KM, Haddad F. Effects of different activity and inactivity paradigms on myosin heavy chain gene expression in striated muscle. J Appl Physiol. 2001;90(1):345-357. doi:10.1152/ jappl.2001.90.1.345

53. Goubert D, De Pauw R, Meeus M, et al. Lumbar muscle structure and function in chronic versus recurrent low back pain: a cross-sectional study. Spine J. 2017;17(9):1285-1296. doi:10.1016/j.spinee.2017.04.025

54. Mannion AF. Fibre type characteristics and function of the human paraspinal muscles: normal values and changes in association with low back pain. $J$ Electromyogr Kinesiol. 1999;9 (6):363-377. doi:10.1016/S1050-6411(99)00010-3

55. D'hooge R, Cagnie B, Crombez G, Vanderstraeten G, Achten E, Danneels L. Lumbar muscle dysfunction during remission of unilateral recurrent nonspecific low-back pain: evaluation with muscle functional MRI. Clin J Pain. 2013;29(3):187-194. doi:10.1097/AJP.0b013e31824ed170

56. Hodges PW, James G, Blomster L, et al. Multifidus muscle changes after back injury are characterized by structural remodeling of muscle, adipose and connective tissue, but not muscle atrophy: molecular and morphological evidence. Spine. 2015;40 (14):1057-1071. doi:10.1097/BRS.0000000000000972

57. Mannion AF, Weber BR, Dvorak J, Grob D, Müntener M. Fibre type characteristics of the lumbar paraspinal muscles in normal healthy subjects and in patients with low back pain. $J$ Orthop Res. 1997;15(6):881-887. doi:10.1002/jor.1100150614

58. Ebenbichler GR, Oddsson LI, Kollmitzer J, Erim Z. Sensorymotor control of the lower back: implications for rehabilitation. Med Sci Sports Exerc. 2001;33(11):1889-1898. doi:10.1097/ 00005768-200111000-00014

59. Freeman MD, Woodham MA, Woodham AW. The role of the lumbar multifidus in chronic low back pain: a review. $P M R$. 2010;2(2):142-146; quiz 1 p following 167. doi:10.1016/j. pmrj.2009.11.006

60. Agten A, Stevens S, Verbrugghe J, Timmermans A, Vandenabeele F. Biopsy samples from the erector spinae of persons with nonspecific chronic low back pain display a decrease in glycolytic muscle fibers. Spine J. 2020;20(2):199-206. doi:10.1016/j.spinee.2019.09.023 
61. Schilder A, Hoheisel U, Magerl W, Benrath J, Klein T, Treede RD. Sensory findings after stimulation of the thoracolumbar fascia with hypertonic saline suggest its contribution to low back pain. Pain. 2014;155(2):222-231. doi:10.1016/j.pain.2013.09.025

62. Shah JP, Gilliams EA. Uncovering the biochemical milieu of myofascial trigger points using in vivo microdialysis: an application of muscle pain concepts to myofascial pain syndrome $J$ Bodyw Mov Ther. 2008;12(4):371-384. doi:10.1016/j. jbmt.2008.06.006

63. Cagnie B, Dewitte V, Barbe T, Timmermans F, Delrue N, Meeus M. Physiologic effects of dry needling. Curr Pain Headache Rep. 2013;17(8):348. doi:10.1007/s11916-013-0348-5

64. Shah JP, Danoff JV, Desai MJ, et al. Biochemicals associated with pain and inflammation are elevated in sites near to and remote from active myofascial trigger points. Arch Phys Med Rehabil. 2008;89(1):16-23. doi:10.1016/j.apmr.2007.10.018

65. Yoshida M, Nakamura T, Sei A, Kikuchi T, Takagi K, Matsukawa A. Intervertebral disc cells produce tumor necrosis factor alpha, interleukin-1beta, and monocyte chemoattractant protein-1 immediately after herniation: an experimental study using a new hernia model. Spine. 2005;30(1):55-61. doi:10.1097/01.brs.0000149194.17891.bf

66. Takada T, Nishida K, Doita M, Miyamoto H, Kurosaka M. Interleukin- 6 production is upregulated by interaction between disc tissue and macrophages. Spine. 2004;29(10):1089-1092; discussion 1093. doi:10.1097/00007632-200405150-00007

67. Kadow T, Sowa G, Vo N, Kang JD. Molecular basis of intervertebral disc degeneration and herniations: what are the important translational questions. Clin Orthop Relat Res. 2015;473 (6):1903-1912. doi:10.1007/s11999-014-3774-8

68. Sunderkötter C, Steinbrink K, Goebeler M, Bhardwaj R, Sorg C. Macrophages and angiogenesis. J Leukoc Biol. 1994;55 (3):410-422. doi:10.1002/jlb.55.3.410

69. Doita M, Kanatani T, Ozaki T, Matsui N, Kurosaka M, Yoshiya S. Influence of macrophage infiltration of herniated disc tissue on the production of matrix metalloproteinases leading to disc resorption. Spine. 2001;26(14):1522-1527. doi:10.1097/ 00007632-200107150-00004

70. Risbud MV, Shapiro IM. Role of cytokines in intervertebral disc degeneration: pain and disc content. Nat Rev Rheumatol. 2014;10 (1):44-56. doi:10.1038/nrrheum.2013.160

71. Johnson WE, Caterson B, Eisenstein SM, Hynds DL, Snow DM, Roberts S. Human intervertebral disc aggrecan inhibits nerve growth in vitro. Arthritis Rheum. 2002;46(10):2658-2664. doi:10.1002/art.10585

72. Nakawaki M, Uchida K, Miyagi M, et al. Changes in nerve growth factor expression and macrophage phenotype following intervertebral disc injury in mice. J Orthop Res. 2019;37 (8):1798-1804. doi:10.1002/jor.24308

73. Krock E, Millecamps M, Currie JB, Stone LS, Haglund L. Low back pain and disc degeneration are decreased following chronic toll-like receptor 4 inhibition in a mouse model. Osteoarthritis Cartilage. 2018;26(9):1236-1246. doi:10.1016/j. joca.2018.06.002

74. Autio RA, Karppinen J, Niinimäki J, et al. Determinants of spontaneous resorption of intervertebral disc herniations. Spine. 2006;31 (11):1247-1252. doi:10.1097/01.brs.0000217681.83524.4a

75. Shamji MF, Setton LA, Jarvis W, et al. Proinflammatory cytokine expression profile in degenerated and herniated human intervertebral disc tissues. Arthritis Rheum. 2010;62(7):1974-1982. doi:10.1002/art.27444

76. Kobayashi S, Meir A, Kokubo Y, et al. Ultrastructural analysis on lumbar disc herniation using surgical specimens: role of neovascularization and macrophages in hernias. Spine. 2009;34 (7):655-662. doi:10.1097/BRS.0b013e31819c9d5b
77. Molinos M, Almeida CR, Caldeira J, Cunha C, Gonçalves RM, Barbosa MA. Inflammation in intervertebral disc degeneration and regeneration. $J R$ Soc Interface. 2015;12(104):20141191. doi:10.1098/rsif.2014.1191

78. Li Y, Liu J, Liu ZZ, Duan DP. Inflammation in low back pain may be detected from the peripheral blood: suggestions for biomarker. Biosci Rep. 2016;36(4). doi:10.1042/BSR20160187

79. van den Berg R, Jongbloed EM, de Schepper E, Bierma-Zeinstra S, Koes BW, Luijsterburg P. The association between pro-inflammatory biomarkers and nonspecific low back pain: a systematic review. Spine J. 2018;18(11):2140-2151. doi:10.1016/j.spinee.2018.06.349

80. Murata Y, Rydevik B, Nannmark U, et al. Local application of interleukin-6 to the dorsal root ganglion induces tumor necrosis factor- $\alpha$ in the dorsal root ganglion and results in apoptosis of the dorsal root ganglion cells. Spine. 2011;36(12):926-932. doi:10.1097/BRS.0b013e3181e7f4a9

81. Trbojević-Akmačić I, Vučković F, Vilaj M, et al. Plasma $\mathrm{N}$-glycome composition associates with chronic low back pain. Biochim Biophys Acta Gen Subj. 2018;1862(10):2124-2133. doi:10.1016/j.bbagen.2018.07.003

82. Diers M, Koeppe C, Diesch E, et al. Central processing of acute muscle pain in chronic low back pain patients: an EEG mapping study. J Clin Neurophysiol. 2007;24(1):76-83. doi:10.1097/01. wnp.0000241093.00844.0e

83. Roussel NA, Nijs J, Meeus M, Mylius V, Fayt C, Oostendorp R. Central sensitization and altered central pain processing in chronic low back pain: fact or myth. Clin J Pain. 2013;29 (7):625-638. doi:10.1097/AJP.0b013e31826f9a71

84. Meints SM, Mawla I, Napadow V, et al. The relationship between catastrophizing and altered pain sensitivity in patients with chronic low-back pain. Pain. 2019;160(4):833-843. doi:10.1097/ j.pain.0000000000001461

85. Iglesias-González JJ, Muñoz-García MT, Rodrigues-de-souza DP, Alburquerque-Sendín F, Fernández-de-las-peñas C. Myofascial trigger points, pain, disability, and sleep quality in patients with chronic nonspecific low back pain. Pain Med. 2013;14 (12):1964-1970. doi:10.1111/pme.12224

86. Niddam DM. Brain manifestation and modulation of pain from myofascial trigger points. Curr Pain Headache Rep. 2009;13 (5):370-375. doi:10.1007/s11916-009-0060-7

87. Hocking MJ. Exploring the central modulation hypothesis: do ancient memory mechanisms underlie the pathophysiology of trigger points. Curr Pain Headache Rep. 2013;17(7):347. doi:10.1007/s11916-013-0347-6

88. Sanzarello I, Merlini L, Rosa MA, et al. Central sensitization in chronic low back pain: a narrative review. J Back Musculoskelet Rehabil. 2016;29(4):625-633. doi:10.3233/BMR-160685

89. Imamura M, Chen J, Matsubayashi SR, et al. Changes in pressure pain threshold in patients with chronic nonspecific low back pain. Spine. 2013;38(24):2098-2107. doi:10.1097/ 01.brs.0000435027.50317.d7

90. Katz WA, Rothenberg R. Section 3: the nature of pain: pathophysiology. J Clin Rheumatol. 2005;11(2 Suppl):S11-15. doi:10.1097/01.rhu.0000158686.43637.af

91. Baranauskas G, Nistri A. Sensitization of pain pathways in the spinal cord: cellular mechanisms. Prog Neurobiol. 1998;54 (3):349-365. doi:10.1016/S0301-0082(97)00067-1

92. Kong J, Spaeth RB, Wey HY, et al. S1 is associated with chronic low back pain: a functional and structural MRI study. Mol Pain. 2013;9:43. doi:10.1186/1744-8069-9-43

93. Letzen JE, Robinson ME. Negative mood influences default mode network functional connectivity in patients with chronic low back pain: implications for functional neuroimaging biomarkers. Pain. 2017;158(1):48-57. doi:10.1097/j.pain.0000000000000708 
94. Yu R, Gollub RL, Spaeth R, Napadow V, Wasan A, Kong J. Disrupted functional connectivity of the periaqueductal gray in chronic low back pain. Neuroimage Clin. 2014;6:100-108. doi:10.1016/j.nicl.2014.08.019

95. Kregel J, Meeus M, Malfliet A, et al. Structural and functional brain abnormalities in chronic low back pain: a systematic review. Semin Arthritis Rheum. 2015;45(2):229-237. doi:10.1016/j. semarthrit.2015.05.002

96. Yarnitsky D. Role of endogenous pain modulation in chronic pain mechanisms and treatment. Pain. 2015;156(Suppl 1):S24-31. doi:10.1097/01.j.pain.0000460343.46847.58

97. McPhee ME, Vaegter HB, Graven-Nielsen T. Alterations in pronociceptive and antinociceptive mechanisms in patients with low back pain: a systematic review with meta-analysis. Pain. 2020;161(3):464-475. doi:10.1097/j.pain.0000000000001737

98. Baliki MN, Petre B, Torbey S, et al. Corticostriatal functional connectivity predicts transition to chronic back pain. Nat Neurosci. 2012;15(8):1117-1119. doi:10.1038/nn.3153

99. Vachon-Presseau E, Tétreault P, Petre B, et al. Corticolimbic anatomical characteristics predetermine risk for chronic pain. Brain. 2016;139(Pt 7):1958-1970. doi:10.1093/brain/aww100

100. Vlaeyen JW, Crombez G, Linton SJ. The fear-avoidance model of pain. Pain. 2016;157(8):1588-1589. doi:10.1097/j. pain.0000000000000574

101. Hashmi JA, Baliki MN, Huang L, et al. Shape shifting pain: chronification of back pain shifts brain representation from nociceptive to emotional circuits. Brain. 2013;136(Pt 9):2751-2768. doi:10.1093/brain/awt211

102. Loggia ML, Chonde DB, Akeju O, et al. Evidence for brain glial activation in chronic pain patients. Brain. 2015;138(Pt 3):604-615. doi:10.1093/brain/awu377

103. Zhang J, Shi XQ, Echeverry S, Mogil JS, De Koninck Y, Rivest S. Expression of CCR2 in both resident and bone marrow-derived microglia plays a critical role in neuropathic pain. $J$ Neurosci. 2007;27(45):12396-12406. doi:10.1523/ JNEUROSCI.3016-07.2007

104. Verma V, Sheikh Z, Ahmed AS. Nociception and role of immune system in pain. Acta Neurol Belg. 2015;115(3):213-220. doi:10.1007/s13760-014-0411-y

105. Hayashida K, Nanki T, Girschick H, Yavuz S, Ochi T, Lipsky PE. Synovial stromal cells from rheumatoid arthritis patients attract monocytes by producing MCP-1 and IL-8. Arthritis Res. 2001;3 (2):118-126. doi:10.1186/ar149

106. Palada V, Ahmed AS, Finn A, Berg S, Svensson CI, Kosek E. Characterization of neuroinflammation and periphery-to-CNS inflammatory cross-talk in patients with disc herniation and degenerative disc disease. Brain Behav Immun. 2019;75:60-71. doi:10.1016/j.bbi.2018.09.010

107. Ehrlich LC, Hu S, Sheng WS, et al. Cytokine regulation of human microglial cell IL-8 production. J Immunol. 1998;160 (4):1944-1948.
108. Koyama Y, Kotani M, Sawamura T, Kuribayashi M, Konishi R, Michinaga S. Different actions of endothelin-1 on chemokine production in rat cultured astrocytes: reduction of CX3CL1/fractalkine and an increase in CCL2/MCP-1 and CXCL1/CINC-1. J Neuroinflammation. 2013;10:51. doi:10.1186/1742-2094-10-51

109. DeLeo JA, Winkelstein BA. Physiology of chronic spinal pain syndromes: from animal models to biomechanics. Spine. 2002;27 (22):2526-2537. doi:10.1097/00007632-200211150-00026

110. Lim T, Anderson KM, Hari P, et al. Evidence for a role of nerve injury in painful intervertebral disc degeneration: a cross-sectional proteomic analysis of human cerebrospinal fluid. J Pain. 2017;18(10):1253-1269. doi:10.1016/j.jpain.2017.06.002

111. Echeverry S, Shi XQ, Rivest S, Zhang J. Peripheral nerve injury alters blood-spinal cord barrier functional and molecular integrity through a selective inflammatory pathway. J Neurosci. 2011;31 (30):10819-10828. doi:10.1523/JNEUROSCI.1642-11.2011

112. Lim TK, Shi XQ, Martin HC, et al. Blood-nerve barrier dysfunction contributes to the generation of neuropathic pain and allows targeting of injured nerves for pain relief. Pain. 2014;155 (5):954-967. doi:10.1016/j.pain.2014.01.026

113. Müller-Schwefe G, Morlion B, Ahlbeck K, et al. Treatment for chronic low back pain: the focus should change to multimodal management that reflects the underlying pain mechanisms. Curr Med Res Opin. 2017;33(7):1199-1210. doi:10.1080/ 03007995.2017.1298521

114. Chung JW, Zeng Y, Wong TK. Drug therapy for the treatment of chronic nonspecific low back pain: systematic review and meta-analysis. Pain Physician. 2013;16(6):E685-704. doi:10.36076/ppj.2013/16/E685

115. Kivitz AJ, Gimbel JS, Bramson C, et al. Efficacy and safety of tanezumab versus naproxen in the treatment of chronic low back pain. Pain. 2013;154(7):1009-1021. doi:10.1016/j. pain.2013.03.006

116. Maher C, Underwood M, Buchbinder R. Non-specific low back pain. Lancet. 2017;389(10070):736-747. doi:10.1016/S01406736(16)30970-9

117. Stynes S, Konstantinou K, Dunn KM. Classification of patients with low back-related leg pain: a systematic review. $B M C$ Musculoskelet Disord. 2016;17:226. doi:10.1186/s12891-0161074-z

118. Saragiotto BT, Maher CG, Moseley AM, et al. A systematic review reveals that the credibility of subgroup claims in low back pain trials was low. J Clin Epidemiol. 2016;79:3-9. doi:10.1016/j.jclinepi.2016.06.003

119. Deyo RA, Von Korff M, Duhrkoop D. Opioids for low back pain. BMJ. 2015;350:g6380. doi:10.1136/bmj.g6380

Journal of Pain Research

\section{Dovepress}

\section{Publish your work in this journal}

The Journal of Pain Research is an international, peer reviewed, open access, online journal that welcomes laboratory and clinical findings in the fields of pain research and the prevention and management of pain. Original research, reviews, symposium reports, hypothesis formation and commentaries are all considered for publication. The manuscript management system is completely online and includes a very quick and fair peer-review system, which is all easy to use. Visit http:// www.dovepress.com/testimonials.php to read real quotes from published authors. 